\title{
Prognostic Role of Tumor-Infiltrating Lymphocytes in Lung Cancer: a Meta- Analysis
}

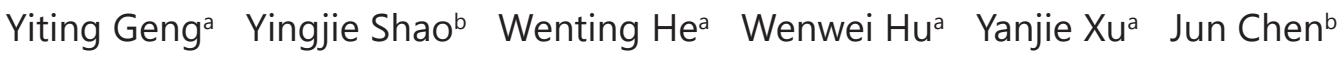 \\ Changping $\mathrm{Wu}^{\mathrm{a}}$ Jingting Jiang ${ }^{\mathrm{a}}$ \\ aDepartment of Oncology, The Third Affiliated Hospital of Soochow University, Changzhou, \\ bDepartment of Radiation Oncology, The Third Affiliated Hospital of Soochow University, Changzhou, \\ P.R. China
}

\section{Key Words}

Tumor-infiltrating lymphocytes $•$ Lung Cancer • Prognosis • Meta-analysis

\begin{abstract}
Background/Aims: The role of Tumor-infiltrating lymphocytes (TILs) in the prognosis of patients with lung cancer is still controversial. We performed a meta-analysis to evaluate the prognostic role of TILs in lung cancer. Methods: Studies were recruited by searching PubMed, Embase and the Cochrane Library and assessed by further quality evaluation. The pooled hazard ratios (HRs) with 95\% confidence intervals (CIs) were calculated to investigate the association between TIL subsets and lung cancer patients' outcome. Results: A total of 29 articles including 8,600 patients were enrolled into the meta-analysis. Our results indicated that high level of $\mathrm{CD}^{+}$cells infiltration in tumor stroma (TS) or tumor nest (TN) was associated with better OS in lung cancer patients ( $\mathrm{HR}=0.76,95 \% \mathrm{CI} 0.62-0.93, P=0.006 ; \mathrm{HR}=0.80,95 \%$ CI 0.67-0.96; $P=0.018$, respectively). Similar results could be also observed in $\mathrm{CD}^{+} \mathrm{T}$ cells infiltration. High $\mathrm{CD}^{+} \mathrm{T}$ lymphocytes infiltration in TS was explicitly accompanied by better OS (HR $=0.65,95 \% \mathrm{CI} 0.46-0.91 ; P=0.013)$, rather than in TN. In contrast, high density of FOXP3 $^{+}$T cells infiltration in TS showed a poor PFS (HR $\left.=2.67,95 \% \mathrm{CI}, 1.74-4.08, P<0.001\right)$. Conclusion: This meta-analysis clarified that high level of $\mathrm{CD}^{+}$and $\mathrm{CD}^{+}{ }^{+}$cells infiltration in TS or TN, and high CD4+ $\mathrm{T}$ lymphocytes infiltration in TS showed better OS in lung cancer patients, whereas high density of FOXP3 ${ }^{+} \mathrm{T}$ cells infiltration in TS could be recognized as a negative prognostic factor.
\end{abstract}




\section{Cellular Physiology Cell Physiol Biochem 2015;37:1560-1571

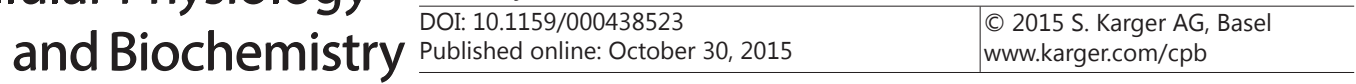 \\ Geng et al.: Prognostic Value of TILs in Lung Cancer}

\section{Introduction}

As a malignant tumor with the highest morbidity and mortality, lung cancer has become a major public health problem worldwide [1]. With the development of the comprehensive treatment strategies, the therapeutic effect of lung cancer has been improved recently. However, the long-term survival of patients is still poor, with a 5-year survival rate of around $18 \%$ [2]. Traditionally, the prognosis of lung cancer was evaluated according to TNM staging based on anatomy and histopathology, which has been proved not accurately enough by growing evidence nowadays. Therefore, a new biological marker is needed for more accurate prognosis and effective therapies as a complement to the TNM system.

Tumor-infiltrating lymphocytes (TILs) might be one of potential biomarkers for cancer prognosis. Early in 1922, McCarfy et al. [3] brought up the concept of TILs, and considered the infiltration of lymphocytes into tumor tissue as an antitumor activity of immune system. Unexpectedly, in the late 1990s, the infiltration of neoplastic tissues by immune inflammatory cells was reported to promote tumor progression. Until recently, a bidirectional role of TILs within the tumor microenvironment in tumor progression has been confirmed: It can not only suppress tumor growth by destroying cancer cells or inhibiting their outgrowth, but also promote tumor progression either by selecting for tumor cells that are more fit to survive in an immunocompetent host or by establishing conditions within the tumor microenvironment that facilitate tumor outgrowth [4]. TILs are a heterogeneous population comprising mainly $\mathrm{T}$ lymphocytes, and to a lesser degree, B lymphocytes and natural killer cells (NK) [5]. It is important to discuss TILs subsets separately due to their different physiological and pathological effects in tumor microenvironment. With the development of immunohistochemistry, more and more subtypes of TILs have been discovered. CD3, a biomarker of T lymphocytes, is expressed in almost all T lymphocytes. According to the cell surface markers, $\mathrm{T}$ lymphocytes mainly include the following subtypes: $\mathrm{CD}^{+}$cytotoxic $\mathrm{T}$ lymphocytes (CTL), CD4 $4^{+} \mathrm{T}$ helper lymphocytes (Th), CD45RO ${ }^{+}$memory $\mathrm{T}$ cells (Tm), and FOXP3 ${ }^{+}$regulatory cells (Tregs), etc.

The correlation between TILs and clinical outcome have been investigated in many cancers, such as lung cancer [6], colorectal cancer [7, 8], breast cancer [9], melanoma [10], ovarian cancer [11], pancreatic cancer [12] and so on. In some studies, TILs were found to be a better predictor of patients' survival than TNM staging [6-9]. However, the prognostic value of TILs in lung cancer is still controversial, varying with the distribution site and cell types. It was reported that high density of $\mathrm{CD}^{+} \mathrm{T}$ cells in tumor stroma (TS) was associated with a longer overall survival (OS) [6], whereas Kawai et al. considered that only CD8 ${ }^{+} \mathrm{T}$ cells in tumor nest (TN) were associated with patients' survival [13]. However, Goc et al. reported that the level of $\mathrm{CD}^{+} \mathrm{T}$ cells in both $\mathrm{TN}$ and $\mathrm{TS}$ had prognostic value for lung cancer patients [14]. These controversial results were also reported in $\mathrm{CD}^{+} \mathrm{T}$ cells $[15,16]$. Compared to $\mathrm{CD}^{+}$and $\mathrm{CD}^{+} \mathrm{T}$ cells, regulatory $\mathrm{T}$ cells $\left(\mathrm{Foxp}^{+}\right.$) showed the opposite effect on prognosis in most studies [17-19]. All the published literatures we have searched so far did not come to a conclusion whether TILs can be used as a potent biomarker for prognosis. Therefore, a systematical and comprehensive meta-analysis to investigate the relationship between TILs and the survival of patients with lung cancer is urgently required. To our knowledge, this meta-analysis is the first to evaluate the prognostic value of TILs in lung cancer patients.

\section{Materials and Methods}

We carried out this meta-analysis following the guidelines of the Systematic Reviews and MetaAnalyses (PRISMA) [20] and the Observational Studies in Epidemiology (MOOSE) guidelines [21].

Search strategy

Literatures were searched through PubMed, Embase and the Cochrane Library (last update by Aug 20, 2015). Keywords used in the search strategy were "tumor-infiltrating lymphocyte OR TILs OR tumor 


\section{Cellular Physiology Cell Physiol Biochem 2015;37:1560-1571

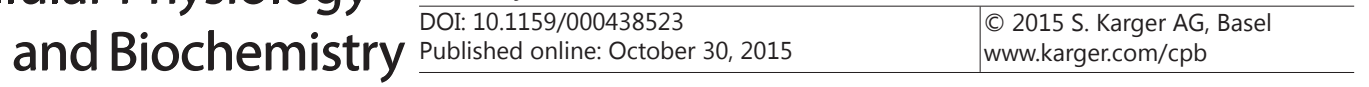 \\ Geng et al.: Prognostic Value of TILs in Lung Cancer}

infiltrating lymphocyte OR intratumoural lymphocyte OR intratumoral lymphocyte OR intra-tumoral lymphocyte" (all fields) AND "lung" (all fields) AND "tumor OR tumour OR neoplasm OR cancer OR carcinoma" (all fields) AND "prognosis OR prognostic OR survival OR outcome" (all fields). There were no other limitations in the database searching process. Besides title, abstract and full text, all the reference lists of identified articles were also reviewed in order to find out potential studies. Two authors were responsible for the comprehensive database search and availability evaluation independently (Y. Geng and Y. Shao).

\section{Inclusion and exclusion criteria}

Literatures that were eligible for inclusion in this meta-analysis met the following criteria: (1) Studies in lung cancer reporting the prognostic impact of TILs or associated TILs subsets (including CD3 ${ }^{+}, \mathrm{CD}^{+}$, $\mathrm{CD}^{+}$, and FoxP3 ${ }^{+}$lymphocytes); (2) Studies provided sufficient data to estimate the hazard ratio (HR) and 95\% confidence intervals (CI) were included in the meta-analyses; (3) The sample size of studies was specific restricted: only studies with sample size $\geq 50$ were enrolled in this meta-analyses. The exclusion criteria were as follows: (1) Studies with sample size $<50$ were exclude, because too small sample size would induce publication bias, whereas if the lower limit was too large, there would be few qualified studies left; (2) If more than one study were targeted at the same patient cohort, only the most recent or complete study would be selected; (3) Some styles of literature such as case reports, letters, reviews, conference abstracts and animal trials were excluded.

\section{Data extraction and quality assessment}

Parameters of all eligible studies was collected, including first author's surname, publication year, origin of population, sample size, tumor stage, lymph node metastasis, follow-up period, TILs subsets and distribution site, the cut-off definition, HRs of TILs and each TILs subset for OS, disease-free survival (DFS) and relapse-free survival (PFS) as well as corresponding 95\% CIs. If univariate and multivariate analyses were both involved in a study, only the latter was selected due to its high precision.

The quality of each study was assessed independently by two researchers according to the NewcastleOttawa Quality Assessment Scale (NOS) [22]. Scores ranged from 0 to 9 for quality assessment, and studies with scores $\geq 6$ were rated as high quality.

\section{Statistical analysis}

The death risk of lung cancer patients with different level of TILs was evaluated by HRs and 95\% CIs: an observed HR > 1 indicated worse prognosis in patients with high-density TILs and a HR $<1$ suggested better prognosis. If the statistical variables were not reported in the article directly, we calculated them from available numerical data in the articles according to the methods described by Tierney [23]. The data from Kaplan-Meier survival curves were read by three independent researchers using Engauge Digitizer version 4.1 to reduce reading variability, and the specific method was described in our previous study [24]. We obtained additional information and original data needed for meta-analysis by sending e-mail to the corresponding authors of eligible articles. Statistical heterogeneity was assessed by visual inspection of forest plots, by performing the Chi-square test (assessing the $P$ value), and by calculating the $I^{2}$ statistic $[25,26]$. If the $P$ value was less than 0.05 and/or $I^{2}$ exceeded $50 \%$, indicating the presence of heterogeneity, a random-effects model (the DerSimonian-Laird method) would be applied. Otherwise, the fixed-effects model (the Mante-Haenszel method) was used. Subgroup analysis and meta-regression were further performed to explore the source of identified heterogeneity. Publication bias was estimated by visually assessing the asymmetry of an inverted funnel plot, and was quantified by Begg's and Egger's tests. For all analyses, STATA version 12.0 (Stata Corporation, College Station, TX, USA) was used, with significance defined as a $P$-value less than 0.05 .

\section{Results}

\section{Study characteristics}

Using the described searching strategy, 962 references were initially retrieved. After screening the titles, abstracts, publication types and full text of each publication, 40 articles investigated the correlation between TILs and patients' outcome in lung cancer. Among these, 
11 articles were excluded Fig. 1. Flow diagram of the study selection process.

(seven without some important data, one investigating the same patient cohorts with others, and three with too small sample size). Finally, 29 articles were enrolled into the meta-analysis (Fig. 1) [6, 1319, 27-47]. The total number of patients from Japan, America, France, Norway, Korea, Greece, Denmark, China, Germany, Austria, Italy, Finland and England was 8,600, ranging from 56 to 1290 patients per study. Six studies included fewer than 100 patients and 15 studies enrolled over 200 patients.

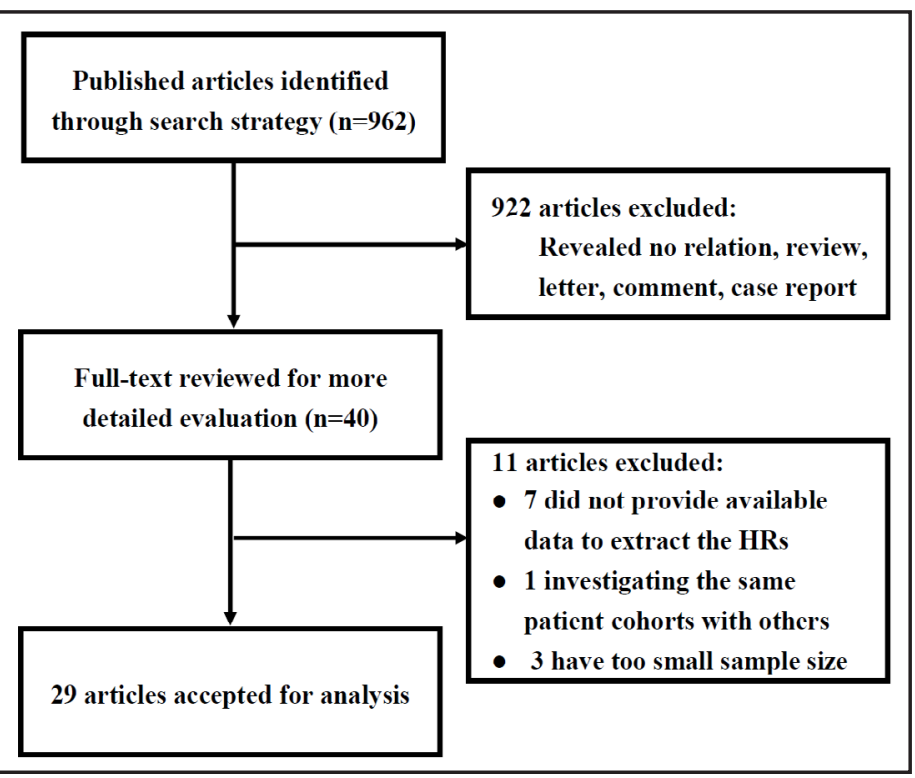

Among these, only four articles reported the prognostic value of generalized TILs [27, $31,38,45]$, and others focused on specific TILs subsets. HE (hematoxylin-eosin) staining and immunohistochemistry (IHC) staining were applied for the detection of generalized TILs and specific TILs subsets respectively. The cut-off values contained median level $(n=11)$, mean level $(n=2)$, and some semiquantitative methods. The other major details of these eligible studies, such as sex, tumor stage, follow-up time, etc, are showed in Table 1.

Quality assessment was performed for each study included in our meta-analyses according to the NOS, with scores ranging from 4 to 8 (mean $=6.7$ ). A higher value indicated a better methodology. Therefore, all these 29 studies were enrolled in the subsequent analyses.

\section{Subgroup analysis}

The prognosis of lung cancer patients is significantly associated with the cell type and distribution site of TILs. Thus, we performed subgroup analyses bases on TILs subsets, and then based on distribution site of each subset. The main results of subgroup analyses are summarized in Table 2.

\section{Generalized TILS}

Three articles researched the relation between the density of generalized TILs in both TN and TS and patients' survival [27, 31, 38, 45]. Three of the articles evaluated OS [27, $38,45]$ and two of them evaluated PFS [27, 38]. Our results showed that high density of generalized TILs was associated with a favorable PFS in lung cancer (the pooled $H R=0.42$, 95\% CI 0.28-0.61, $P<0.001)$, rather than OS (HR $=0.95,95 \%$ CI 0.77-1.18, $P=0.66)$.

\section{CD8 $8^{+}$Tlymphocyte subset}

A total of 18 articles, encompassing 6,543 tumor patients, focused on the association between the infiltration of CD8 $8^{+} \mathrm{T}$ lymphocytes and the survival of lung cancer patients $[6$, $13,14,28-30,32-34,38-40,42-47]$. Stratified by the distribution of TILs, there were 11 of TN, 6 of TS and 6 of both TN and TS, respectively.

\section{Tumor nest}

Ten studies $[13,14,29,30,32,33,39,40,42,44]$ which assessed the infiltration of CD8 ${ }^{+}$ $\mathrm{T}$ lymphocytes in TN were involved in OS analysis, with a significant heterogeneity among them $\left(P=0.002, I^{2}=66.4 \%\right)$. Hence, a random model was applied to overall data integration, and the result suggested that patients with high $\mathrm{CD} 8^{+} \mathrm{T}$ lymphocytes infiltration had better 


\begin{tabular}{|c|c|c|}
\hline Cellular Physiology & Cell Physiol Biochem 2015;37:1560-1571 & \\
\hline and Biochemistry & $\begin{array}{l}\text { DOI: 10.1159/000438523 } \\
\text { Published online: October 30, } 2015\end{array}$ & $\begin{array}{l}\text { O } 2015 \mathrm{~S} \text {. Karger AG, Basel } \\
\text { www.karger.com/cpb }\end{array}$ \\
\hline
\end{tabular}

Table 1. Main characteristics of all studies included in the meta-analysis. TS tumor stroma, TN tumor nest, NR not report, IHC immunohistochemistry, HE haematoxylin-eosin, HPV high-power fields, OS overall survival, PFS recurrence-free survival, DFS disease-free survival, DSS disease-special survival, QIF quantitative immunofluorescence

\begin{tabular}{|c|c|c|c|c|c|c|c|c|c|}
\hline Study & Subset & Location & $\begin{array}{l}\text { Origin of } \\
\text { population }\end{array}$ & $\begin{array}{l}\text { Sample number } \\
\text { (male/female) }\end{array}$ & $\begin{array}{l}\text { Tumor stage } \\
\text { ( I / II / III /IV) }\end{array}$ & $\begin{array}{l}\text { Follow-up } \\
\text { (months) }\end{array}$ & $\begin{array}{c}\text { Detected } \\
\text { method }\end{array}$ & $\begin{array}{l}\text { Cut-off for } \\
\text { overexpression }\end{array}$ & Outcome \\
\hline \multirow[t]{2}{*}{ Goc 2014 [14] } & $\mathrm{CD}^{+}$ & TS & France & $376(302 / 74)$ & $167 / 101 / 104 / 2$ & $\geq 90$ & $\mathrm{IHC}$ & 383 per $\mathrm{mm}^{2}$ & OS \\
\hline & $\mathrm{CD}^{+}$ & TN & France & $376(302 / 74)$ & $167 / 101 / 104 / 2$ & $\geq 90$ & $\mathrm{IHC}$ & 114 per $\mathrm{mm}^{2}$ & os \\
\hline Kinoshita 2013 [17] & FOXP3+ $^{+}$ & TS & Japan & $200(93 / 107)$ & $200 / 0 / 0 / 0$ & $3-87$ & IHC & mean $(6 / \mathrm{HPF})$ & OS, PFS \\
\hline \multirow[t]{2}{*}{ Liu 2012 [28] } & $\mathrm{CD}^{+}+$ & both & China & $259(118 / 41)$ & $0 / 0 / 96 / 63$ & median $20.8(2-44)$ & IHC & median & os \\
\hline & FOXP3+ & both & China & $259(118 / 41)$ & $0 / 0 / 96 / 63$ & median $20.8(2-44)$ & IHC & median & os \\
\hline \multirow[t]{2}{*}{ Kayser 2012 [15] } & $\mathrm{CD}^{+}+$ & TS & Germany & $232(167 / 65)$ & $91 / 59 / 77 / 3$ & median 32(1-210) & IHC & median 74.6 per $\mathrm{mm}^{2}$ & os \\
\hline & $\mathrm{CD}^{+}+$ & $\mathrm{TN}$ & Germany & $232(167 / 65)$ & $91 / 59 / 77 / 3$ & median 32(1-210) & IHC & median 19.8 per $\mathrm{mm}^{2}$ & os \\
\hline Ilie 2012 [29] & $\mathrm{CD}^{+}$ & $\mathrm{TN}$ & France & $632(467 / 165)$ & $274 / 185 / 173 / 0$ & median $30(0-112)$ & IHC & median 110 per $\mathrm{mm}^{2}$ & OS \\
\hline \multirow[t]{3}{*}{ Sterlacci 2012 [30] } & $\mathrm{CD} 4^{+}$ & $\mathrm{TN}$ & Austria & $383(280 / 103)$ & $219 / 67 / 79 / 18$ & over 60 & IHC & mean $16.84 / 0.28 \mathrm{~mm}^{2}$ & OS \\
\hline & $\mathrm{CD}^{+}$ & $\mathrm{TN}$ & Austria & $383(280 / 103)$ & $219 / 67 / 79 / 18$ & over 60 & IHC & mean $17.92 / 0.28 \mathrm{~mm}^{2}$ & os \\
\hline & FOXP3+ & $\mathrm{TN}$ & Austria & $383(280 / 103)$ & $219 / 67 / 79 / 18$ & over 60 & IHC & mean $1.17 / 0.28 \mathrm{~mm}^{2}$ & os \\
\hline Tao 2012 [18] & $\mathrm{FOXP}^{+}$ & TS & Japan & $87(56 / 31)$ & $\mathrm{NR}$ & $\mathrm{NR}$ & $\mathrm{IHC}$ & $25 / \mathrm{HPF}$ & OS, PFS \\
\hline Horne 2011 [27] & TIL & both & American & $273(130 / 143)$ & $273 / 0 / 0 / 0$ & NR & $\mathrm{HE}$ & moderate lymphocytes & OS, PFS \\
\hline \multirow[t]{2}{*}{ Al-shibli 2010 [16] } & $\mathrm{CD}^{+}$ & TS & Norway & $335(253 / 82)$ & $212 / 91 / 32 / 0$ & median $86(48-216)$ & IHC & $50 \%$ & os \\
\hline & $\mathrm{CD}^{+}$ & $\mathrm{TN}$ & Norway & $253 / 82(335)$ & $212 / 91 / 32 / 0$ & median $86(48-216)$ & $\mathrm{IHC}$ & $1 \%$ & OS \\
\hline Kilic 2011 [31] & TIL & both & American & $219(\mathrm{NR} / \mathrm{NR})$ & $219 / 0 / 0 / 0$ & over24 & $\mathrm{HE}$ & none to mild infiltrate & PFS \\
\hline Ruffini 2009 [32] & $\mathrm{CD}^{+}$ & $\mathrm{TN}$ & Italy & $1290(1084 / 206)$ & $714 / 265 / 214 / 0$ & $\mathrm{NR}$ & IHC & staining in $20 \%$ of cells & os \\
\hline \multirow[t]{3}{*}{ Al-shibli 2008 [6] } & $\mathrm{CD}^{+}+$ & TS & Norway & $335(253 / 82)$ & $212 / 91 / 32 / 0$ & median $96(10-179)$ & $\mathrm{IHC}$ & $25 \%$ & DSS \\
\hline & $\mathrm{CD}^{+}+$ & $\mathrm{TN}$ & Norway & $335(253 / 82)$ & $212 / 91 / 32 / 0$ & median $96(10-179)$ & IHC & $5 \%$ & DSS \\
\hline & $\mathrm{CD} 8^{+}$ & $\mathrm{TN}$ & Norway & $335(253 / 82)$ & $212 / 91 / 32 / 0$ & median $96(10-179)$ & IHC & $5 \%$ & DSS \\
\hline \multirow[t]{2}{*}{ Kawai 2008 [13] } & $\mathrm{CD}^{+}$ & $\mathrm{TN}$ & Japan & $199(139 / 60)$ & $0 / 0 / 0 / 199$ & $\mathrm{NR}$ & $\mathrm{IHC}$ & median $12 / \mathrm{HPV}$ & os \\
\hline & $\mathrm{CD}^{+}$ & TS & Japan & $199(139 / 60)$ & $0 / 0 / 0 / 199$ & NR & IHC & median $13 / \mathrm{HPV}$ & os \\
\hline \multirow[t]{2}{*}{ Wakabayashi 2003 [33] } & $\mathrm{CD}^{+}+$ & TS & Japan & $144(97 / 47)$ & I88/II-III56 & over 36 & IHC & median $210 / \mathrm{HPV}$ & os \\
\hline & $\mathrm{CD}^{+}+$ & $\mathrm{TN}$ & Japan & $178(118 / 60)$ & I107/II-III71 & over 36 & $\mathrm{IHC}$ & median 35/HPV & os \\
\hline \multirow[t]{2}{*}{ Hiraoka 2006 [34] } & $\mathrm{CD}^{+}+$ & TS & Japan & $109(71 / 38)$ & I67/II-III42 & over 60 & IHC & median $168 / \mathrm{HPV}$ & os \\
\hline & $\mathrm{CD}^{+}$ & TS & Japan & $109(71 / 38)$ & I67/II-III42 & over 60 & IHC & median $151 / \mathrm{HPV}$ & os \\
\hline Shimizu 2010 [35] & FOXP3+ & TS & Japan & $100(60 / 40)$ & $68 / 14 / 18 / 0$ & over 24 & IHC & median $3 / \mathrm{HPV}$ & PFS \\
\hline Petersen 2006 [36] & $\mathrm{CD}^{+}$ & both & American & $64(34 / 30)$ & $64 / 0 / 0 / 0$ & over 30 & IHC & $50 \%$ & PFS \\
\hline \multirow[t]{2}{*}{ Suzuki 2013 [19] } & Foxp3+ & TS & American & $478(176 / 302)$ & $478 / 0 / 0 / 0$ & over 24 & IHC & 1.67 normal & PFS \\
\hline & Foxp $3^{+}$ & TS & American & $478(186 / 292)$ & $478 / 0 / 0 / 0$ & over 24 & $\mathrm{IHC}$ & 1.67 normal & PFS \\
\hline Dieu-Nosjean 2008 [37] & $\mathrm{CD}^{+}+$ & both & France & $74(60 / 14)$ & $62 / 12 / 0 / 0$ & over 48 & IHC & 1.5 mean score/tumor IPF & os \\
\hline \multirow[t]{2}{*}{ Ikeda 2006 [38] } & $\mathrm{CD} 8^{+}$ & both & Japan & $83(65 / 18)$ & I40/II-III 43 & over 60 & $\mathrm{IHC}$ & $5 / \mathrm{HPV}$ & OS \\
\hline & TIL & both & Japan & $83(65 / 18)$ & I40/II-III 43 & over 60 & $\mathrm{HE}$ & $50 / \mathrm{HPV}$ & os \\
\hline Yoshida 2006 [39] & $\mathrm{CD}^{+}$ & $\mathrm{TN}$ & Japan & $125(86 / 39)$ & $71 / 26 / 25 / 2$ & median 21.7 & $\mathrm{IHC}$ & median 220/HPV & os \\
\hline Eerola 2000 [40] & $\mathrm{CD} 8^{+}$ & $\mathrm{TN}$ & Finland & $56(51 / 5)$ & I-II37/III-IV19 & over 60 & $\mathrm{IHC}$ & median $10 / \mathrm{HPV}$ & os \\
\hline Johnson 2000 [41] & $\mathrm{CD}^{+}$ & $\mathrm{TN}$ & England & $95(59 / 36)$ & $54 / 17 / 20 / 0$ & $\mathrm{NR}$ & $\mathrm{IHC}$ & $10 / \mathrm{HPV}$ & os \\
\hline \multirow[t]{2}{*}{ Kikuchi 2007 [42] } & $\mathrm{CD}^{+}$ & TN & Japan & $161(110 / 51)$ & 195/II-III-IV66 & median 111.7 & IHC & median & OS \\
\hline & $\mathrm{CD}^{+}$ & TS & Japan & $161(110 / 51)$ & I95/II-III-IV66 & median 111.7 & IHC & median & os \\
\hline Donnem 2015 [43] & $\mathrm{CD} 8^{+}$ & TS & Norway Dermark & $797(510 / 287)$ & $407 / 275 / 115 / 0$ & median 65.5 & IHC & $50 \%$ & OS, DFS, DSS \\
\hline Alifano 2014 [44] & $\mathrm{CD}^{+}$ & TN & France & $303(244 / 59)$ & $114 / 85 / 97 / 7$ & NR & IHC & 96 per $\mathrm{mm}^{2}$ & os \\
\hline \multirow[t]{6}{*}{ Schalper 2015 [45] } & TIL & both & American & $188(91 / 97)$ & I-II119/III-IV55 & NR & HE & the top tertile & os \\
\hline & TIL & both & Greece & $341(300 / 41)$ & I-II200/III-IV135 & NR & HE & $60 \%$ & os \\
\hline & $\mathrm{CD}^{+}+$ & both & American & $188(91 / 97)$ & I-II119/III-IV55 & NR & QIF & $60 \%$ & os \\
\hline & $\mathrm{CD}^{+}$ & both & Greece & $341(300 / 41)$ & I-II200/III-IV135 & NR & QIF & the top tertile & os \\
\hline & $\mathrm{CD} 8^{+}$ & both & American & $188(91 / 97)$ & I-II119/III-IV55 & NR & QIF & the top tertile & os \\
\hline & $\mathrm{CD} 8^{+}$ & both & Greece & $341(300 / 41)$ & I-II200/III-IV135 & NR & QIF & the top tertile & os \\
\hline Kim 2015 [46] & $\mathrm{CD}^{+}$ & both & Korea & $331(317 / 14)$ & $131 / 118 / 79 / 0$ & NR & $\mathrm{IHC}$ & median 251.5 per $\mathrm{mm}^{2}$ & OS, DFS \\
\hline Koh 2015 [47] & $\mathrm{CD} 8^{+}$ & both & Korea & $497(231 / 266)$ & $342 / 46 / 109 / 0$ & NR & IHC & 100 per $\mathrm{mm}^{2}$ & DFS \\
\hline
\end{tabular}

OS (HR=0.80, 95\% CI 0.67-0.96; $P=0.018$ ) (Fig. 2A). In order to explore the potential factors responsible for the heterogeneity, we conducted subgroup analyses, sensitivity analysis and meta-regression. The subgroups were defined according to the main features of pooled studies, including cut-off values, follow-up time, publication year, patients' ethnicity, and sample size. We found that high level of $\mathrm{CD}^{+} \mathrm{T}$ lymphocytes was associated with improved OS in groups with Caucasian patients(HR $=0.83,95 \%$ CI 0.75-0.92; $P<0.001)$, special cutoff values (non-median; HR $=0.75,95 \%$ CI 0.58-0.96; $P=0.024$ ), long time follow-up ( $\geq 60$ months; HR $=0.76,95 \%$ CI 0.64-0.90; $P=0.002$ ), more recent publication (after 2009; HR $=0.84,95 \%$ CI $0.75-0.93 ; P=0.001)$ and large sample size $(\geq 300$; HR $=0.84,95 \%$ CI $0.75-$ $0.93 ; P=0.001$ ); However, statistical significance was not shown in other subgroups (Table $3)$. Sensitivity analysis was performed by sequential omission of individual studies using the fixed-effects model, and the result pattern was not obviously impacted by any single study. The meta-regression showed that the cut-off values $(P=0.646)$, follow-up time $(P=0.390)$, publication year $(P=0.803)$, patients' ethnicity $(P=0.572)$ and sample size $(P=0.803)$ may contributed to the heterogeneity more or less, but not definitively. 


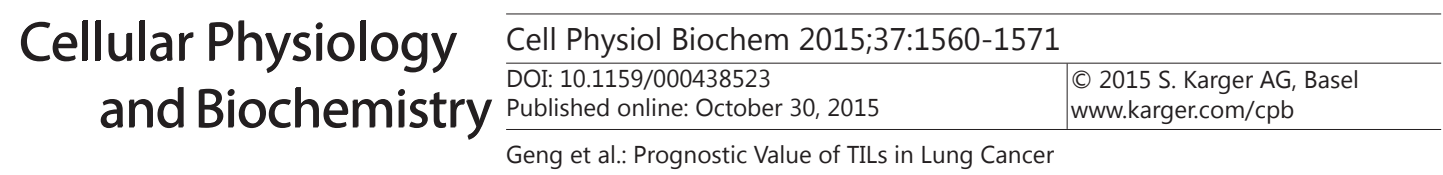

Table 2. The pooled associations between TILs subsets and the prognosis of patients with lung cancer. TS tumor stroma, TN tumor nest, OS overall survival, PFS recurrence-free survival, DSS disease-special survival, HR hazard ratio, CI confidence intervals

\begin{tabular}{|c|c|c|c|c|c|c|c|c|c|}
\hline \multirow{2}{*}{ Subset/distribution } & \multirow{2}{*}{ Outcome } & \multirow{2}{*}{ No. of studies } & \multirow{2}{*}{ No. of patients } & \multirow{2}{*}{ HR $(95 \% \mathrm{CI})$ - model } & \multirow{2}{*}{$P$ value } & \multicolumn{2}{|c|}{ heterogeneity } & \multicolumn{2}{|c|}{ Publication bias } \\
\hline & & & & & & $I^{2}(\%)$ & $P$ & Begg's $P$ & Egger's $P$ \\
\hline \multicolumn{10}{|l|}{ Generalized TILs } \\
\hline \multirow{2}{*}{ both } & os & 4 & 885 & $0.95(0.77-1.18)$ - fixed & 0.66 & 0 & 0.521 & 0.734 & 0.818 \\
\hline & PFS & 2 & 492 & $0.42(0.28-0.61)-$ fixed & $<0.001^{\circ}$ & 0 & 0.409 & - & - \\
\hline \multicolumn{10}{|l|}{$\mathrm{CD}^{+}+$} \\
\hline \multirow[t]{2}{*}{$\mathrm{TN}$} & os & 10 & 3703 & $0.80(0.67-0.96)$ - random & $0.018^{*}$ & 66.4 & 0.002 & 0.371 & 0.366 \\
\hline & DSS & 1 & 335 & $0.67(0.46-0.97)$ & $0.023^{*}$ & - & - & - & - \\
\hline \multirow[t]{3}{*}{ TS } & os & 5 & 1642 & $0.76(0.62-0.93)$ - random & $0.006^{*}$ & 63.4 & 0.027 & 0.462 & 0.856 \\
\hline & DFS & 1 & 797 & $0.72(0.62-0.85)$ & $<0.001^{\circ}$ & - & - & - & - \\
\hline & DSs & 1 & 797 & $0.49(0.30-0.82)$ & $<0.001^{\circ}$ & - & - & - & - \\
\hline \multirow[t]{2}{*}{ both } & os & 5 & 1102 & $0.74(0.63-0.88)$ - fixed & $<0.001^{\circ}$ & 0 & 0.406 & 0.806 & 0.261 \\
\hline & DFS & 2 & 828 & $0.68(0.55-0.85)-$ fixed & $0.001^{*}$ & 0 & 0.523 & - & - \\
\hline \multicolumn{10}{|l|}{$\mathrm{CD}^{+}+$} \\
\hline $\mathrm{TN}$ & os & 3 & 662 & $0.66(0.45-0.97)$ - random & $0.036^{*}$ & 60.1 & 0.082 & 1.000 & 0.636 \\
\hline TS & os & 2 & 567 & $0.65(0.50-0.84)-$ fixed & $0.001^{*}$ & 0 & 0.487 & - & - \\
\hline \multirow[t]{2}{*}{ both } & os & 3 & 603 & $0.72(0.51-1.03)$ - random & 0.068 & 53.4 & 0.093 & 1 & 0.327 \\
\hline & PFS & 2 & 138 & $0.50(0.10-2.42)-$ random & 0.392 & 84.4 & 0.011 & - & - \\
\hline \multicolumn{10}{|l|}{$\mathrm{CD}^{+}+$} \\
\hline \multirow[t]{2}{*}{$\mathrm{TN}$} & os & 2 & 508 & $0.43(0.07-2.61)$ - random & 0.359 & 92.9 & 0.001 & - & - \\
\hline & DSS & 1 & 335 & $0.94(0.64-1.37)$ & 0.632 & - & - & - & - \\
\hline \multirow[t]{2}{*}{ TS } & os & 2 & 253 & $0.65(0.46-0.91)-$ fixed & $0.013^{*}$ & 0 & 0.395 & - & - \\
\hline & DSS & 1 & 335 & $0.42(0.20-0.59)$ & $<0.001^{\circ}$ & - & - & - & - \\
\hline \multicolumn{10}{|l|}{ FOXP3 ${ }^{+}$} \\
\hline \multirow[t]{2}{*}{ TS } & os & 2 & 287 & $2.67(1.74-4.08)$ - fixed & $<0.001^{\circ}$ & 0 & 0.829 & - & - \\
\hline & PFS & 5 & 1343 & $2.14(1.68-2.72)-$ fixed & $<0.001^{\circ}$ & 0 & 0.898 & 1.000 & 0.879 \\
\hline $\mathrm{TN}$ & os & 1 & 383 & $0.92(0.39-2.17)$ & 0.849 & - & - & - & - \\
\hline both & os & 1 & 159 & $1.22(0.91-1.64)$ & 0.186 & - & - & - & - \\
\hline
\end{tabular}

Table 3. Subgroup analyses of the relationship between $\mathrm{CD}^{+} \mathrm{T}$ lymphocyte subsets and OS. OS overall survival, HR hazard ratio, CI confidence intervals

\begin{tabular}{|c|c|c|c|c|c|c|}
\hline \multirow{2}{*}{ Outcome subgroup } & \multirow{2}{*}{$\begin{array}{c}\text { No. of } \\
\text { patients }\end{array}$} & \multirow{2}{*}{$\begin{array}{l}\text { No. of } \\
\text { studie: }\end{array}$} & \multirow{2}{*}{ HR (95\%CI) } & \multirow{2}{*}{$P$ value } & \multicolumn{2}{|c|}{ Heterogeneity } \\
\hline & & & & & $I^{2}$ & $P$ \\
\hline Overall effect & 3703 & 10 & $0.80(0.67-0.96)$ - random & $0.018^{*}$ & 66.4 & 0.002 \\
\hline \multicolumn{7}{|l|}{ Patients' ethnicity } \\
\hline Asian & 663 & 4 & $0.91(0.58-1.43)$ - random & 0.677 & 83.9 & $<0.001$ \\
\hline Caucasian & 3040 & 6 & $0.83(0.75-0.92)-$ fixed & $<0.001^{*}$ & 37.6 & 0.156 \\
\hline \multicolumn{7}{|l|}{ cut-off values } \\
\hline median & 1351 & 6 & $0.85(0.64-1.13)$ - random & 0.261 & 75.7 & 0.001 \\
\hline others & 2352 & 4 & $0.75(0.58-0.96)$ - random & $0.024^{*}$ & 50.7 & 0.108 \\
\hline \multicolumn{7}{|l|}{ Follow-up months } \\
\hline$\geq 60$ & 1279 & 5 & $0.76(0.64-0.90)-$ fixed & $0.002^{*}$ & 38.0 & 0.168 \\
\hline$<60$ & 2424 & 5 & $0.89(0.69-1.15)$ - random & 0.357 & 78.6 & 0.001 \\
\hline \multicolumn{7}{|l|}{ Publication year } \\
\hline Before 2009 & 719 & 5 & $0.83(0.55-1.27)$ - random & 0.398 & 80.5 & $<0.001$ \\
\hline After 2009 & 2984 & 5 & $0.84(0.75-0.93)-$ fixed & $0.001^{*}$ & 36.3 & 0.179 \\
\hline \multicolumn{7}{|l|}{ Sample size } \\
\hline$\geq 300$ & 2984 & 5 & $0.84(0.75-0.93)-$ fixed & $0.001^{*}$ & 36.3 & 0.425 \\
\hline$<300$ & 719 & 5 & $0.83(0.55-1.27)$ - random & 0.398 & 80.5 & $<0.001$ \\
\hline
\end{tabular}

\section{Tumor stroma}

As regards the 5 studies providing OS [13, 14, 34, 42, 43], a random model was used to calculate the pooled HR and its $95 \% \mathrm{CI}$ due to the high heterogeneity between these studies $\left(P=0.027, I^{2}=63.4 \%\right)$. The pooled HR indicated that high level of $\mathrm{CD}^{+} \mathrm{T}$ lymphocytes in TS significantly predicted better OS (HR=0.76, 95\% CI 0.62-0.93; $P=0.006)$ (Fig. 2A).

\section{Both tumor nest and stroma}

Five studies researched the $\mathrm{CD}^{+} \mathrm{T}$ lymphocytes in both TN and TS [28, 38, 45-47]. Because these studies did not display obvious heterogeneity $\left(P=0.406, I^{2}=0 \%\right)$, a fixed model was used for calculating the pooled HR. Our result showed that high level of CD8 ${ }^{+}$ T lymphocytes in both TN and TS is a favorable predictor for OS (HR=0.74, 95\% CI 0.63-0.88; $P<0.001$ ) and DFS (HR=0.68, 95\% CI 0.55-0.85; $P<0.001$ ) (Fig. $2 \mathrm{~A}$ ). 


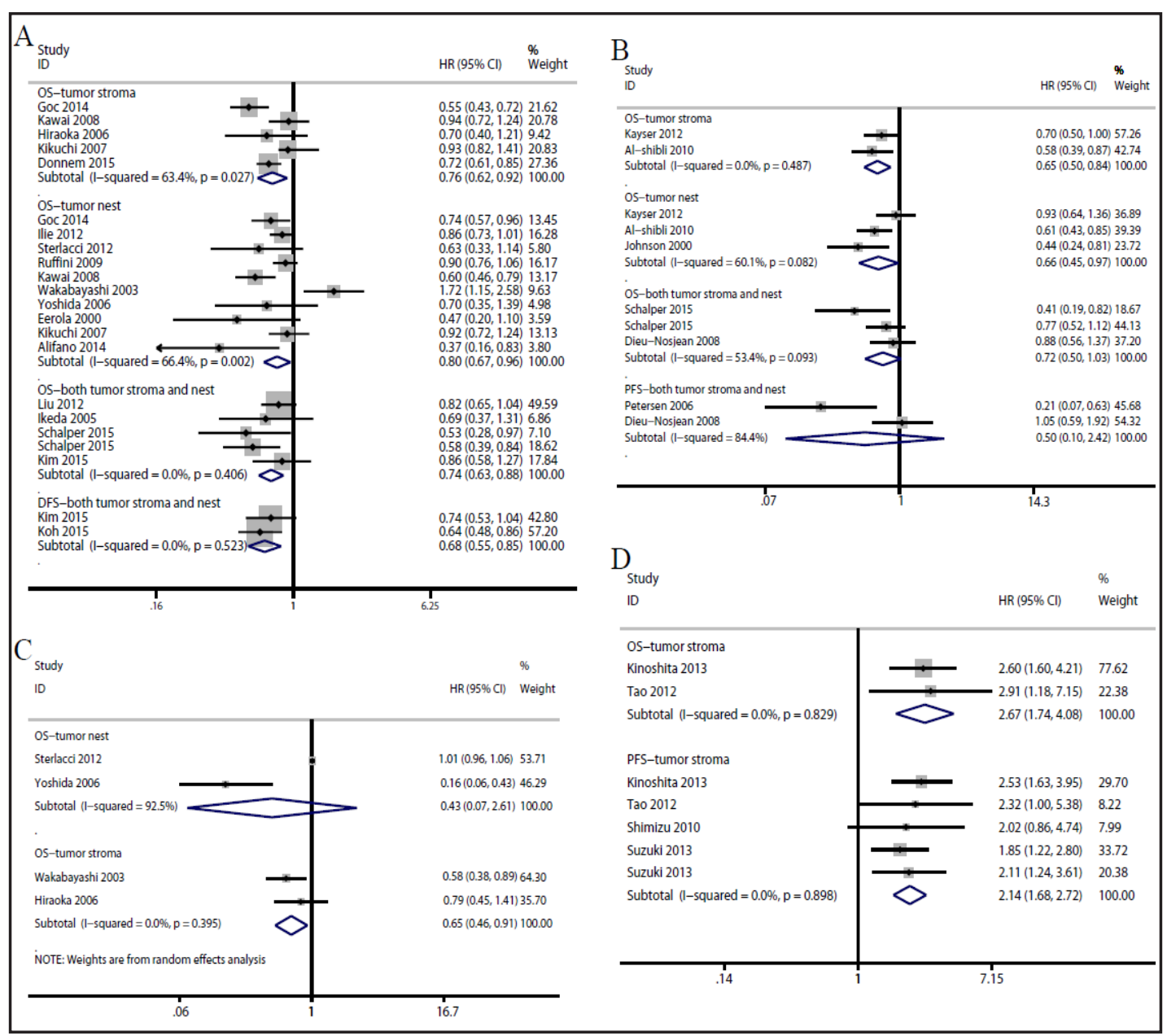

Fig. 2. Forest plots of studies evaluating hazard ratios of high level of TILs subsets in lung cancer by the distribution. (A) Forest plots of the prognostic effect of $\mathrm{CD} 8^{+} \mathrm{T}$ cells. (B) Forest plots of prognostic effect of $\mathrm{CD}^{+} \mathrm{T}$ cells. (C) Forest plots of prognostic effect of $\mathrm{CD} 4^{+} \mathrm{T}$ cells. (D) Forest plots of prognostic effect of $\mathrm{FOXP}^{+} \mathrm{T}$ cells.

Fig. 3. Funnel plots of $\mathrm{CD}^{+} \mathrm{T}$ cell infiltration in tumor nest.

CD3 ${ }^{+}$T lymphocyte subset

Six articles provided data regarding the association between $\mathrm{CD}^{+}{ }^{+} \mathrm{T}$ cells infiltration and survival outcomes [15, 16, 36, 37, 41, 45]. Three of them $[15,16,41]$ investigated $\mathrm{CD}^{+} \mathrm{T}$ cells infiltration in $\mathrm{TN}$, and the pooled HR for OS was

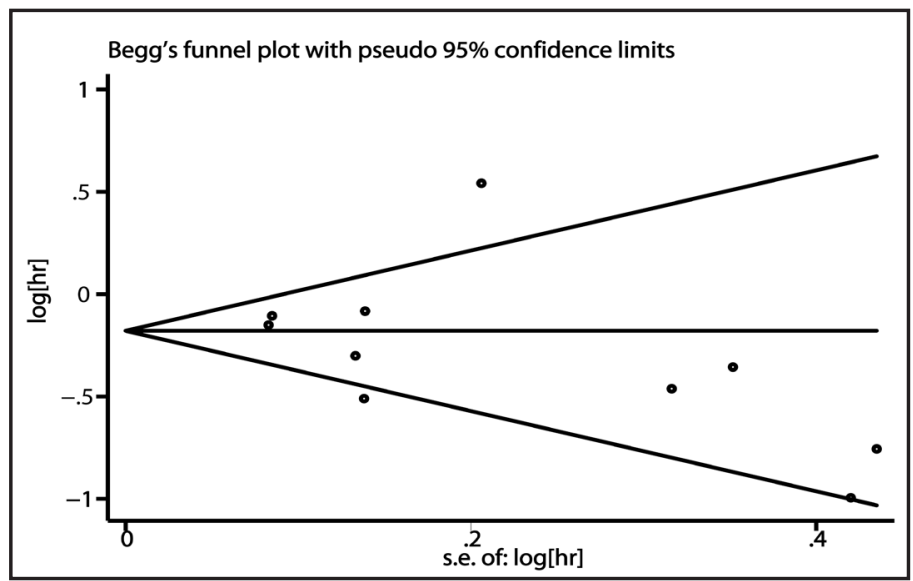
0.66 (95\% CI $0.45-0.97 ; P=0.036)$, with a significant evidence of heterogeneity $(P=0.082$, $I^{2}=60.1 \%$ ) (Fig. 2B). Two studies which focused on $\mathrm{CD}^{+} \mathrm{T}$ cells infiltration in TS $[15,16]$ confirmed a positive predictive effect for OS (HR=0.65, 95\% CI 0.50-0.84; $P=0.001$ ) without heterogeneity (Fig. 2B). However, high $\mathrm{CD}^{+} \mathrm{T}$ cells infiltration in both $\mathrm{TN}$ and $\mathrm{TS}$ did not show a positive outcome for both OS and PFS (Fig. 2B). 


\section{Cellular Physiology Cell Physiol Biochem 2015;37:1560-1571 \begin{tabular}{l|l} 
and Biochemistry Published online: October 30, 2015 & $\begin{array}{l}\text { C } 2015 \text { S. Karger AG, Basel } \\
\text { www.karger.com/cpb }\end{array}$ \\
\hline
\end{tabular} \\ Geng et al.: Prognostic Value of TILs in Lung Cancer}

CD4 $4^{+}$T lymphocyte subset

Five eligible articles [6, 30, 33, 34, 39] provided the HR and 95\% CI regarding the correlation between $\mathrm{CD} 4^{+} \mathrm{T}$ cells and lung cancer patients' survival. We found that high $\mathrm{CD} 4^{+}$ T lymphocytes infiltration in TS was explicitly accompanied by better OS (HR $=0.65,95 \%$ CI 0.46-0.91; $P=0.013$ ), rather than in TN (HR=0.43, 95\% CI 0.07-2.61; $P=0.359$ ) (Fig. 2C).

\section{$\mathrm{FOXP3}^{+}$Treg subset}

Six articles researched the prognostic value of FOXP3 ${ }^{+}$Tregs in lung cancer patients $[17-19,28,30,35]$. Five studies investigated FOXP3 ${ }^{+}$Tregs in TS [17-19, 35], and the pooled HRs for OS and PFS were 2.67 (95\% CI, 1.74-4.08) and 2.14 (95\% CI, 1.68-2.72), respectively, without any heterogeneity (Fig. 2D). Thus, it is reliable that high level of FOXP3 ${ }^{+}$Tregs in TS is associated with poor outcome in lung cancer. There was no prognostic effect of FOXP3 ${ }^{+}$ Tregs in TN [30] both TN and TS [28], with only one study in each group.

Publication Bias

The publication bias of all enrolled studies was evaluated using Egger's and Begg's tests. In subgroup analyses, the $P$ values of Egger's and Begg's tests were all greater than 0.05 (Table 2). Additionally, a funnel plot was applied to detect publication bias for the subgroups with the largest number of studies. The funnel plot of the $\mathrm{CD}^{+} \mathrm{T}$ cells infiltration in TN was substantially symmetric (Fig. 3). Therefore, significant publication bias was not observed in our meta-analyses.

\section{Discussion}

The TNM staging system has been used for over 80 years, but provides incomplete prognostic information. Clinical outcome may vary widely among patients within the same histological tumor stage [48]. Recently, a combination of the density and location of TILs subsets has defined the Immunoscore as a complement to the TNM system for the classification of malignancy [49-51]. Although derived from the immune contexture [52,53], Immunoscore is more convenient to evaluated than the immune contexture. In colorectal cancer, the Immunoscore is a simple and powerful prognostic biomarker: it has been confirmed significant prognostic value even in Cox multivariate including TNM classification $[7,8]$. However, this concept is not well explored in lung cancer. It's timely to definitively clarity which subset or location of TILs is appropriate for lung cancer Immunoscore. We conducted a meta-analysis combining 29 studies and 8,600 patients to provide clinical evidence for Immunoscore in lung cancer. The statistical results confirmed that high density of generalized TILs was associated with favorable PFS, rather than OS. Subgroup analysis was performed according to TILs subsets including $\mathrm{CD}^{+}, \mathrm{CD}^{+}, \mathrm{CD}^{+}$and $\mathrm{FOXP}^{+} \mathrm{T}$ cells, and we found better OS in patients with high level of $\mathrm{CD}^{+} \mathrm{T}$ cells infiltration in TS, TN, and in both TS and TN. Compared with CD8 ${ }^{+} \mathrm{T}$ cells in TN, the prognostic effect of CD8 ${ }^{+} \mathrm{T}$ cells in TS appeared more significant. However, the positive prognostic value of $\mathrm{CD}^{+}{ }^{+} \mathrm{T}$ cells was only found in TS and TN, but not in both TS and TN. High density of CD4 ${ }^{+} \mathrm{T}$ cells infiltration in TS, rather than in TN, was associated with better prognosis in lung cancer. However, high density of FOXP3 ${ }^{+} \mathrm{T}$ cells infiltration in TS could be recognized as a negative prognostic factor. These results were interpreted to indicate that: firstly, various TILs subsets and distribution sites were generally associated with outcome of patients, but the pooled HRs didn't show dramatic differences in survival. Combining different TILs subsets, especially those have opposite effects on survival, would be helpful for prediction of prognosis, such as $\mathrm{CD}^{+}$and $\mathrm{FOXP3}^{+}$, $\mathrm{CD}^{+}$and $\mathrm{FOXP}^{+}, \mathrm{CD}^{+}$and FOXP3 ${ }^{+} \mathrm{T}$ cells. Secondly, it is essential to stratify each subset of $\mathrm{T}$ lymphocytes according to the infiltration location, and the prognostic effect of TILs in TS seemed to be superior to those in TN. This conclusion is based on some physiological theory. Tumor cells use the deregulation of the Fas/Fas ligand (FasL) signaling pathway to escape the immune reactions directed against them. By downregulation of Fas and upregulation of FasL lung cancer cells may escape from cytotoxic T-cell effects and induced apoptosis [54]. 


\section{Cellular Physiology Cell Physiol Biochem 2015;37:1560-1571 \begin{tabular}{ll|l} 
DOI: 10.1159/000438523 & $\begin{array}{l}\text { O 2015 S. Karger AG, Basel } \\
\text { www.karger.com/cpb }\end{array}$ \\
\hline
\end{tabular} \\ Geng et al.: Prognostic Value of TILs in Lung Cancer}

As FasL concentrations will be highest in between tumor cell and thus in the "intraepithelial" compartment, changes of Fas/FasL ratios can lead to inactivation of T-cells, especially the intraepithelial $\mathrm{CD}^{+}$and $\mathrm{CD}^{+}$lymphocytes [15]. The native TILs in both TN and TS express high level of PD-1, connected with PD-L1 which causes anergy of TILs [55]. Cancer associated fibroblasts (CAF) is reported to induce the extravasation of active T cells with PD-1 low expression from the circulatory system via upregulating transforming growth factor (TGF)- $\beta$ and vascular endothelial growth factor (VEGF), which lead to more comprehensive immune response in TS $[17,56,57]$. Additionally, the density of $\mathrm{CD}^{+}$cell infiltrating into cancer nests was decreased in tumor with negative expression of human leukocyte antigen (HLA) class I, but the density of $\mathrm{CD}^{+}$cells infiltrating in stroma was unaffected by expression of HLA class I. Down-regulation of HLA class I expression was a poor prognostic factor in many tumor including NSCLC [42]. Thus, it is reasoned that the anti-tumor immune response mainly depends on the TILs in TS, and the number and function of TILs in TS may particularly influence patients' prognosis. This gives an explanation of our results showing TILs in TS to be of statistically significant prognostic value compared with TILs in TN. In view of the standpoints aforementioned, we propounded the Immunoscore in lung cancer based on the combination of different TILs $\left(\mathrm{CD}^{+}\right.$and $\mathrm{FOXP}^{+}{ }^{+}$cells, $\mathrm{CD}^{+}$and $\mathrm{FOXP}^{+}, \mathrm{CD}^{+}$and FOXP3 $\left.{ }^{+}\right)$ in TS, which is helpful to predict prognosis more accurately. Recently, a research of Suzuki et al. showed that the combination of stromal $\mathrm{CD}^{+}$and $\mathrm{FOXP}^{+} \mathrm{T}$ cells acted as an independent prognostic factor in lung cancer [19]. However, further prospective studies with large sample size are needed to find the most promising combination of TILs for the establishment of Immunoscore in lung cancer.

Dialectically, these results should be carefully understood for following reasons. First, different cut-off points were used in those studies, and the deficiency of a unified cut-off value may affect the precision of TILs as a predictive biomarker in cancer prognosis. Second, several HRs were calculated based on the data extracted from the survival curves, which inevitably leads to small statistical errors. Third, TILs in tumor nest and stroma were discussed in this study. However, because many of the included studies did not address the location and several scores were based on a mix of cores located in the central of the tumor and in the invasive margin, we didn't carry out specific discussion about the detailed localization: central tumor versus invasive margin. Fourth, other subsets of TILs such as B lymphocyte were not included in this research owing to few investigations. Finally, although we performed subgroup analyses pertaining to prognostic association of subsets and distribution of TILs, there was still significant heterogeneity displayed in our meta-analysis, which was most likely due to variation in patient origin, publication year, tumor pathological type, tumor stage, sample size, follow-up time, and cut-off values among others.

\section{Conclusion}

In conclusion, we found that high level of $\mathrm{CD}^{+}$and $\mathrm{CD}^{+} \mathrm{T}$ cells infiltration in TS or TN show better OS in lung cancer patients, whereas high density of FOXP3 ${ }^{+} \mathrm{T}$ cells infiltration in TS could be recognized as a negative prognostic factor. Considering the limitations of present analysis, the conclusions should be regarded cautiously. Further prospective multi-center studies with larger sample size are needed to test the most promising combination of TILs for the establishment of Immunoscore in lung cancer.

\section{Acknowledgements}

This research project was supported by the National Natural Science Foundation of China (NSFC) (81171653).

\section{Disclosure Statement}

The authors declare that they have no conflict of interest. 


\section{Cellular Physiology Cell Physiol Biochem 2015;37:1560-1571 \begin{tabular}{l|l|l} 
DOI: 10.1159/000438523 & (C) 2015 S. Karger AG, Basel
\end{tabular} \\ Geng et al.: Prognostic Value of TILs in Lung Cancer}

\section{References}

1 Jemal A, Bray F, Center MM, Ferlay J, Ward E, Forman D: Global cancer statistics. CA Cancer J Clin 2011;61:69-90.

2 Siegel RL, Miller KD, Jemal A: Cancer statistics, 2015. CA Cancer J Clin 2015;65:5-29.

3 McCarty WC: Principles of prognosis in cancer. JAMA 1931;96:30-33.

4 Schreiber RD, Old LJ, Smyth MJ: Cancer immunoediting: integrating immunity's roles in cancer suppression and promotion. Science 2011;331:1565-1570.

5 Balch CM, Riley LB, Bae YJ, Salmeron MA, Platsoucas CD, von Eschenbach A, Itoh K: Patterns of human tumor-infiltrating lymphocytes in 120 human cancers. Arch Surg 1990;125:200-205.

6 Al-Shibli KI, Donnem T, Al-Saad S, Persson M, Bremnes RM, Busund LT: Prognostic effect of epithelial and stromal lymphocyte infiltration in non-small cell lung cancer. Clin Cancer Res 2008;14:5220-5227.

7 Galon J, Costes A, Sanchez-Cabo F, Kirilovsky A, Mlecnik B, Lagorce-Pages C, Tosolini M, Camus M, Berger A, Wind P, Zinzindohoue F, Bruneval P, Cugnenc PH, Trajanoski Z, Fridman WH, Pages F: Type, density, and location of immune cells within human colorectal tumors predict clinical outcome. Science 2006;313:19601964.

8 Mlecnik B, Tosolini M, Kirilovsky A, Berger A, Bindea G, Meatchi T, Bruneval P, Trajanoski Z, Fridman WH, Pages F, Galon J: Histopathologic-based prognostic factors of colorectal cancers are associated with the state of the local immune reaction. J Clin Oncol 2011;29:610-618.

9 Mahmoud SM, Paish EC, Powe DG, Macmillan RD, Grainge MJ, Lee AH, Ellis IO, Green AR: Tumor-infiltrating CD8+ lymphocytes predict clinical outcome in breast cancer. J Clin Oncol 2011;29:1949-1955.

10 Mackensen A, Ferradini L, Carcelain G, Triebel F, Faure F, Viel S, Hercend T: Evidence for in situ amplification of cytotoxic T-lymphocytes with antitumor activity in a human regressive melanoma. Cancer Res 1993;53:3569-3573.

11 Zhang L, Conejo-Garcia JR, Katsaros D, Gimotty PA, Massobrio M, Regnani G, Makrigiannakis A, Gray H, Schlienger K, Liebman MN, Rubin SC, Coukos G: Intratumoral T cells, recurrence, and survival in epithelial ovarian cancer. N Engl J Med 2003;348:203-213.

12 Fukunaga A, Miyamoto M, Cho Y, Murakami S, Kawarada Y, Oshikiri T, Kato K, Kurokawa T, Suzuoki M, Nakakubo Y, Hiraoka K, Itoh T, Morikawa T, Okushiba S, Kondo S, Katoh H: CD8+ tumor-infiltrating lymphocytes together with CD4+ tumor-infiltrating lymphocytes and dendritic cells improve the prognosis of patients with pancreatic adenocarcinoma. Pancreas 2004;28:e26-31.

13 Kawai O, Ishii G, Kubota K, Murata Y, Naito Y, Mizuno T, Aokage K, Saijo N, Nishiwaki Y, Gemma A, Kudoh S, Ochiai A: Predominant infiltration of macrophages and CD8(+) T Cells in cancer nests is a significant predictor of survival in stage IV nonsmall cell lung cancer. Cancer 2008;113:1387-1395.

14 Goc J, Germain C, Vo-Bourgais TK, Lupo A, Klein C, Knockaert S, de Chaisemartin L, Ouakrim H, Becht E, Alifano M, Validire P, Remark R, Hammond SA, Cremer I, Damotte D, Fridman WH, Sautes-Fridman C, Dieu-Nosjean MC: Dendritic cells in tumor-associated tertiary lymphoid structures signal a Th1 cytotoxic immune contexture and license the positive prognostic value of infiltrating CD8+ T cells. Cancer Res 2014;74:705-715.

15 Kayser G, Schulte-Uentrop L, Sienel W, Werner M, Fisch P, Passlick B, Zur Hausen A, Stremmel C: Stromal CD4/CD25 positive T-cells are a strong and independent prognostic factor in non-small cell lung cancer patients, especially with adenocarcinomas. Lung Cancer 2012;76:445-451.

16 Al-Shibli K, Al-Saad S, Andersen S, Donnem T, Bremnes RM, Busund LT: The prognostic value of intraepithelial and stromal CD3-, CD117- and CD138-positive cells in non-small cell lung carcinoma. APMIS 2010;118:371-382.

17 Kinoshita T, Ishii G, Hiraoka N, Hirayama S, Yamauchi C, Aokage K, Hishida T, Yoshida J, Nagai K, Ochiai A: Forkhead box P3 regulatory T cells coexisting with cancer associated fibroblasts are correlated with a poor outcome in lung adenocarcinoma. Cancer Sci 2013;104:409-415.

18 Tao H, Mimura Y, Aoe K, Kobayashi S, Yamamoto H, Matsuda E, Okabe K, Matsumoto T, Sugi K, Ueoka H: Prognostic potential of FOXP3 expression in non-small cell lung cancer cells combined with tumorinfiltrating regulatory T cells. Lung Cancer 2012;75:95-101.

19 Suzuki K, Kadota K, Sima CS, Nitadori J, Rusch VW, Travis WD, Sadelain M, Adusumilli PS: Clinical impact of immune microenvironment in stage I lung adenocarcinoma: tumor interleukin-12 receptor beta2 (IL12Rbeta2), IL-7R, and stromal FoxP3/CD3 ratio are independent predictors of recurrence. J Clin Oncol 2013;31:490-498.

20 Liberati A, Altman DG, Tetzlaff J, Mulrow C, Gotzsche PC, Ioannidis JP, Clarke M, Devereaux PJ, Kleijnen J, Moher D: The PRISMA statement for reporting systematic reviews and meta-analyses of studies that 


\section{Cellular Physiology Cell Physiol Biochem 2015;37:1560-1571 \begin{tabular}{l|l|l} 
DOI: 10.1159/000438523 & (C) 2015 S. Karger AG, Basel
\end{tabular} \\ Geng et al.: Prognostic Value of TILs in Lung Cancer}

evaluate health care interventions: explanation and elaboration. PLoS Med 2009;6:e1000100.

21 Stroup DF, Berlin JA, Morton SC, Olkin I, Williamson GD, Rennie D, Moher D, Becker BJ, Sipe TA, Thacker SB: Meta-analysis of observational studies in epidemiology: a proposal for reporting. Meta-analysis Of Observational Studies in Epidemiology (MOOSE) group. JAMA 2000;283:2008-2012.

22 Stang A: Critical evaluation of the Newcastle-Ottawa scale for the assessment of the quality of nonrandomized studies in meta-analyses. Eur J Epidemiol 2010;25:603-605.

23 Tierney JF, Stewart LA, Ghersi D, Burdett S, Sydes MR: Practical methods for incorporating summary timeto-event data into meta-analysis. Trials 2007;8:16.

24 Shao Y, Geng Y, Gu W, Huang J, Pei H, Jiang J: Prognostic role of tissue and circulating microRNA-200c in malignant tumors: a systematic review and meta-analysis. Cell Physiol Biochem 2015;35:1188-1200.

25 Higgins JP, Thompson SG: Quantifying heterogeneity in a meta-analysis. Stat Med 2002;21:1539-1558.

26 Higgins JP, Thompson SG, Deeks JJ, Altman DG: Measuring inconsistency in meta-analyses. BMJ 2003;327:557-560.

27 Horne ZD, Jack R, Gray ZT, Siegfried JM, Wilson DO, Yousem SA, Nason KS, Landreneau RJ, Luketich JD, Schuchert MJ: Increased levels of tumor-infiltrating lymphocytes are associated with improved recurrencefree survival in stage 1A non-small-cell lung cancer. J Surg Res 2011;171:1-5.

28 Liu H, Zhang T, Ye J, Li H, Huang J, Li X, Wu B, Huang X, Hou J: Tumor-infiltrating lymphocytes predict response to chemotherapy in patients with advance non-small cell lung cancer. Cancer Immunol Immunother 2012;61:1849-1856.

29 Ilie M, Hofman V, Ortholan C, Bonnetaud C, Coelle C, Mouroux J, Hofman P: Predictive clinical outcome of the intratumoral CD66b-positive neutrophil-to-CD8-positive T-cell ratio in patients with resectable nonsmall cell lung cancer. Cancer 2012;118:1726-1737.

30 Sterlacci W, Wolf D, Savic S, Hilbe W, Schmid T, Jamnig H, Fiegl M, Tzankov A: High transforming growth factor beta expression represents an important prognostic parameter for surgically resected non-small cell lung cancer. Hum Pathol 2012;43:339-349.

31 Kilic A, Landreneau RJ, Luketich JD, Pennathur A, Schuchert MJ: Density of tumor-infiltrating lymphocytes correlates with disease recurrence and survival in patients with large non-small-cell lung cancer tumors. J Surg Res 2011;167:207-210.

32 Ruffini E, Asioli S, Filosso PL, Lyberis P, Bruna MC, Macri L, Daniele L, Oliaro A: Clinical significance of tumor-infiltrating lymphocytes in lung neoplasms. Ann Thorac Surg 2009;87:365-371; discussion 371-362.

33 Wakabayashi O, Yamazaki K, Oizumi S, Hommura F, Kinoshita I, Ogura S, Dosaka-Akita H, Nishimura M: CD4+ T cells in cancer stroma, not CD8+ T cells in cancer cell nests, are associated with favorable prognosis in human non-small cell lung cancers. Cancer Sci 2003;94:1003-1009.

34 Hiraoka K, Miyamoto M, Cho Y, Suzuoki M, Oshikiri T, Nakakubo Y, Itoh T, Ohbuchi T, Kondo S, Katoh H: Concurrent infiltration by CD8 + T cells and CD4+ T cells is a favourable prognostic factor in non-small-cell lung carcinoma. Br J Cancer 2006;94:275-280.

35 Shimizu K, Nakata M, Hirami Y, Yukawa T, Maeda A, Tanemoto K: Tumor-infiltrating Foxp3+ regulatory T cells are correlated with cyclooxygenase-2 expression and are associated with recurrence in resected nonsmall cell lung cancer. J Thorac Oncol 2010;5:585-590.

36 Petersen RP, Campa MJ, Sperlazza J, Conlon D, Joshi MB, Harpole DH Jr, Patz EF Jr: Tumor infiltrating Foxp3+ regulatory T-cells are associated with recurrence in pathologic stage I NSCLC patients. Cancer 2006;107:2866-2872.

37 Dieu-Nosjean MC, Antoine M, Danel C, Heudes D, Wislez M, Poulot V, Rabbe N, Laurans L, Tartour E, de Chaisemartin L, Lebecque S, Fridman WH, Cadranel J: Long-term survival for patients with non-small-cell lung cancer with intratumoral lymphoid structures. J Clin Oncol 2008;26:4410-4417.

38 Ikeda S, Funakoshi N, Inagaki M, Shibata T: Clinicopathologic roles of tumor-infiltrating lymphocytes and CD8-positive lymphocytes in lung cancer imprint smears in squamous cell carcinoma and adenocarcinoma. Acta Cytol 2006;50:423-429.

39 Yoshida N, Abe H, Ohkuri T, Wakita D, Sato M, Noguchi D, Miyamoto M, Morikawa T, Kondo S, Ikeda H, Nishimura T: Expression of the MAGE-A4 and NY-ESO-1 cancer-testis antigens and T cell infiltration in nonsmall cell lung carcinoma and their prognostic significance. Int J Oncol 2006;28:1089-1098.

40 Eerola AK, Soini Y, Paakko P: A high number of tumor-infiltrating lymphocytes are associated with a small tumor size, low tumor stage, and a favorable prognosis in operated small cell lung carcinoma. Clin Cancer Res 2000;6:1875-1881.

41 Johnson SK, Kerr KM, Chapman AD, Kennedy MM, King G, Cockburn JS, Jeffrey RR: Immune cell infiltrates and prognosis in primary carcinoma of the lung. Lung Cancer 2000;27:27-35. 


\section{Cellular Physiology Cell Physiol Biochem 2015;37:1560-1571 \begin{tabular}{l|l|l} 
DOI: 10.1159/000438523 & (C) 2015 S. Karger AG, Basel
\end{tabular}

42 Kikuchi E, Yamazaki K, Torigoe T, Cho Y, Miyamoto M, Oizumi S, Hommura F, Dosaka-Akita H, Nishimura M: HLA class I antigen expression is associated with a favorable prognosis in early stage non-small cell lung cancer. Cancer Sci 2007;98:1424-1430.

43 Donnem T, Hald SM, Paulsen EE, Richardsen E, Al-Saad S, Kilvaer TK, Brustugun OT, Helland A, LundIversen M, Poehl M, Olsen KE, Ditzel HJ, Hansen O, Al-Shibli K, Kiselev Y, Sandanger TM, Andersen S, Pezzella F, Bremnes RM, Busund LT: Stromal CD8+ T-cell Density-A Promising Supplement to TNM Staging in Non-Small Cell Lung Cancer. Clin Cancer Res 2015;21:2635-2643.

44 Alifano M, Mansuet-Lupo A, Lococo F, Roche N, Bobbio A, Canny E, Schussler O, Dermine H, Regnard JF, Burroni B, Goc J, Biton J, Ouakrim H, Cremer I, Dieu-Nosjean MC, Damotte D: Systemic inflammation, nutritional status and tumor immune microenvironment determine outcome of resected non-small cell lung cancer. PLoS One 2014;9:e106914.

45 Schalper KA, Brown J, Carvajal-Hausdorf D, McLaughlin J, Velcheti V, Syrigos KN, Herbst RS, Rimm DL: Objective measurement and clinical significance of TILs in non-small cell lung cancer. J Natl Cancer Inst DOI:10.1093/jnci/dju435.

46 Kim MY, Koh J, Kim S, Go H, Jeon YK, Chung DH: Clinicopathological analysis of PD-L1 and PD-L2 expression in pulmonary squamous cell carcinoma: Comparison with tumor-infiltrating T cells and the status of oncogenic drivers. Lung Cancer 2015;88:24-33.

47 Koh J, Go H, Keam B, Kim MY, Nam SJ, Kim TM, Lee SH, Min HS, Kim YT, Kim DW, Jeon YK, Chung DH: Clinicopathologic analysis of programmed cell death-1 and programmed cell death-ligand 1 and 2 expressions in pulmonary adenocarcinoma: comparison with histology and driver oncogenic alteration status. Mod Pathol 2015;28:1154-1166.

48 Nagtegaal ID, Quirke P, Schmoll HJ: Has the new TNM classification for colorectal cancer improved care? Nat Rev Clin Oncol 2012;9:119-123.

49 Angell H, Galon J: From the immune contexture to the Immunoscore: the role of prognostic and predictive immune markers in cancer. Curr Opin Immunol 2013;25:261-267.

50 Galon J, Pages F, Marincola FM, Angell HK, Thurin M, Lugli A, Zlobec I, Berger A, Bifulco C, Botti G, Tatangelo F, Britten CM, Kreiter S, Chouchane L, Delrio P, Arndt H, Asslaber M, Maio M, Masucci GV, Mihm M, VidalVanaclocha F, Allison JP, Gnjatic S, Hakansson L, Huber C, Singh-Jasuja H, Ottensmeier C, Zwierzina H, Laghi L, Grizzi F, Ohashi PS, Shaw PA, Clarke BA, Wouters BG, Kawakami Y, Hazama S, Okuno K, Wang E, O'Donnell-Tormey J, Lagorce C, Pawelec G, Nishimura MI, Hawkins R, Lapointe R, Lundqvist A, Khleif SN, Ogino S, Gibbs P, Waring P, Sato N, Torigoe T, Itoh K, Patel PS, Shukla SN, Palmqvist R, Nagtegaal ID, Wang Y, D'Arrigo C, Kopetz S, Sinicrope FA, Trinchieri G, Gajewski TF, Ascierto PA, Fox BA: Cancer classification using the Immunoscore: a worldwide task force. J Transl Med 2012;10:205.

51 Galon J, Mlecnik B, Bindea G, Angell HK, Berger A, Lagorce C, Lugli A, Zlobec I, Hartmann A, Bifulco C, Nagtegaal ID, Palmqvist R, Masucci GV, Botti G, Tatangelo F, Delrio P, Maio M, Laghi L, Grizzi F, Asslaber M, D'Arrigo C, Vidal-Vanaclocha F, Zavadova E, Chouchane L, Ohashi PS, Hafezi-Bakhtiari S, Wouters BG, Roehrl M, Nguyen L, Kawakami Y, Hazama S, Okuno K, Ogino S, Gibbs P, Waring P, Sato N, Torigoe T, Itoh K, Patel PS, Shukla SN, Wang Y, Kopetz S, Sinicrope FA, Scripcariu V, Ascierto PA, Marincola FM, Fox BA, Pages F: Towards the introduction of the 'Immunoscore' in the classification of malignant tumours. J Pathol 2014;232:199-209.

52 Galon J, Fridman WH, Pages F: The adaptive immunologic microenvironment in colorectal cancer: a novel perspective. Cancer Res 2007;67:1883-1886.

53 Fridman WH, Pages F, Sautes-Fridman C, Galon J: The immune contexture in human tumours: impact on clinical outcome. Nat Rev Cancer 2012;12:298-306.

54 Viard-Leveugle I, Veyrenc S, French LE, Brambilla C, Brambilla E: Frequent loss of Fas expression and function in human lung tumours with overexpression of FasL in small cell lung carcinoma. J Pathol 2003;201:268-277.

55 Ahmadzadeh M, Johnson LA, Heemskerk B, Wunderlich JR, Dudley ME, White DE, Rosenberg SA: Tumor antigen-specific CD8 T cells infiltrating the tumor express high levels of PD-1 and are functionally impaired. Blood 2009;114:1537-1544.

56 Joyce JA, Fearon DT: T cell exclusion, immune privilege, and the tumor microenvironment. Science 2015;348:74-80.

57 Sun LX, Li WD, Lin ZB, Duan XS, Li XF, Yang N, Lan TF, Li M, Sun Y, Yu M, Lu J: Protection against lung cancer patient plasma-induced lymphocyte suppression by Ganoderma lucidum polysaccharides. Cell Physiol Biochem 2014;33:289-299. 